\title{
FIBROUS AND PROTOPLASMIC ASTROCYTES ARE BIOCHEMICALLY AND DEVELOPMENTALLY DISTINCT ${ }^{1}$
}

\author{
ROBERT H. MILLER AND MARTIN C. RAFF ${ }^{2}$ \\ Medical Research Council Neuroimmunology Project, Department of Zoology, University College London, \\ London WC1E 6BT, England
}

Received July 18, 1983; Revised September 13, 1983; Accepted September 21, 1983

\begin{abstract}
We have studied semithin frozen sections of developing and adult rat central nervous system (CNS) by indirect immunofluorescence in order to determine the antigenic phenotype of protoplasmic and fibrous astrocytes. Using antibodies against glial fibrillary acidic protein (GFAP) to identify astrocytes, we show that the great majority of fibrous astrocytes in adult optic nerve are labeled by the monoclonal antibody A2B5, while the great majority of protoplasmic astrocytes in adult cerebral cortex are not. Astrocytes located at the periphery of the adult optic nerve that form the glial limiting membrane are more like protoplasmic astrocytes than fibrous astrocytes in that they strain relatively weakly with anti-GFAP antiserum and are $\mathrm{A} 2 \mathrm{~B}^{-}$. In the developing rat optic nerve, protoplasmic-like astrocytes appear at least one week before the first fibrous astrocytes can be detected. Taken together with our previous observations on astrocytes in suspensions and cultures of developing rat optic nerve (Raff, M. C., E. R. Abney, J. Cohen, R. Lindsay, and M. Noble (1983) J. Neurosci. 3: 1289-1300; Raff, M. C., R. H. Miller, and M. Noble (1983) Nature 303: $390-396)$, these results suggest that protoplasmic and fibrous astrocytes are distinct classes of glial cells that differ in their antigenic phenotype and developmental history, as well as in their morphology and location within the CNS.
\end{abstract}

Astrocytes contain characteristic intermediate filaments called glial filaments, which are largely polymers of glial fibrillary acidic protein (GFAP) (Bignami et al., 1972; Schachner et al., 1977). The presence of glial filaments is the most reliable criterion for identifying astrocytes in the electron microscope, while the specific decoration of these filaments, by gold in the Cajal gold sublimate stain (Cajal, 1913; Vaughn and Pease, 1967; Mori and Leblond, 1969) or by anti-GFAP antibodies in immunohistochemical procedures (Bignami et al., 1972), provides a reliable way of recognizing such cells in the light microscope.

Two major classes of astrocytes have been distinguished in histological sections of the CNS on the basis of morphology and distribution: fibrous astrocytes have many glial filaments and are located mainly in white

\footnotetext{
${ }^{1}$ We thank Drs. M. Nirenberg and B. Anderton for the A2B5 clone and rabbit anti-NF serum, respectively, and our colleagues for helpful comments on the manuscript. R. H. M. is supported by a fellowship from the National Fund for Research into Crippling Diseases.

${ }^{2}$ 'I'o whom correspondence should be addressed, at MRC Neuroimmunology Project, Department of Zoology, University College London, Gower Street, Iondon WC1E 6BT, England.
}

matter; protoplasmic astrocytes have fewer glial filaments and are found mainly in gray matter (Peters et al., 1976). Whereas fibrous astrocytes tend to have regular contours and to extend cylindrical branching processes, protoplasmic astrocytes have irregular contours and extend sheet-like processes that appear to fill most of the space between the other elements in the neuropil (Peters et al., 1976).

We have recently distinguished two types of astrocytes in cultures of developing rat optic nerve on the basis of morphology, growth characteristics, and labeling with two ligands that bind to polysialogangliosides-tetanus toxin (Van Heyningen, 1963) and the A2B5 monoclonal antibody (Eisenbarth et al., 1979). Type 1 astrocytes have a fibroblast-like morphology, proliferate in culture in response to epidermal growth factor (EGF) or growth factors present in extracts of bovine pituitary, and do not bind detectable amounts of tetanus toxin or A2B5 antibody. Type 2 astrocytes have a process-bearing morphology, resembling neurons or oligodendrocytes, do not proliferate in response to EGF or extracts of bovine pituitary, and bind both tetanus toxin and A2B5 antibody (Raff et al., 1983a). Whereas type 1 astrocytes are found in cultures of both developing white and gray 
matter, type 2 astrocytes are found in cultures of developing white matter but not of developing gray matter (Raff et al., 1983a).

The morphologies and regional origins of type 1 and type 2 astrocytes suggest that these two cell types may correspond to protoplasmic and fibrous astrocytes, respectively. In the experiments to be described, we have explored this possibility further by examining semithin frozen sections (Tokuyasu, 1973) of both developing and adult rat CNS by indirect immunofluorescence to determine the antigenic phenotype of astrocytes in situ. We show that, in adult rats, A2B5 antibody binds to the great majority of fibrous astrocytes in optic nerve but not to most protoplasmic astrocytes in cerebral cortex. Moreover, we demonstrate that the astrocytes that form the glial limiting membrane at the periphery of the adult optic nerve stain less intensely with anti-GFAP antibodies than do the astrocytes in the interior of the nerve and are $\mathrm{A}_{2} \mathrm{B5}^{-}$, suggesting that they are more like protoplasmic than fibrous astrocytes. Finally, we show that, in the developing optic nerve, protoplasmic-like astrocytes appear at least one week before fibrous astrocytes can be detected. We conclude that type 1 and type 2 astrocytes in vitro correspond, respectively, to protoplasmic and fibrous astrocytes in vivo and that these two classes of astrocytes differ in antigenic phenotype and developmental history, as well as in morphology and distribution in the CNS.

\section{Materials and Methods}

Frozen sections. Adult and 1- to 4-week-old SpragueDawley rats were perfused for $5 \mathrm{~min}$ through the ascending aorta with $3 \%$ paraformaldehyde (BDH) and $0.2 \%$ glutaraldehyde (Emscope) in $0.1 \mathrm{M}$ phosphate buffer, $\mathrm{pH}$ 7.4 (buffer). The paraformaldehyde was prepared by heating to $60^{\circ} \mathrm{C}$ in distilled water and adding $\mathrm{NaOH}$ until the solution was clear. In the case of 1-day-old rats, the optic nerves were fixed in situ by flooding the exposed nerves in the same fixative for several hours. The relevant tissues (optic nerves or pieces of cerebral cortex) were removed and immersed in the same fixative for a minimum of $2 \mathrm{hr}$. The tissues were then cut into small pieces of no more than $2 \mathrm{~mm}^{3}$ and immersed in $1 \mathrm{M}$ sucrose $(\mathrm{BDH})$ with $2 \%$ paraformaldehyde in buffer at room temperature for at least $1 \mathrm{hr}$, to allow the sucrose to penetrate the fixed tissue and act as a cryoprotectant (Tokuyasu, 1973). The tissue blocks were mounted in the desired orientation on the stub of a freezing microtome and frozen by rapid immersion into a slush of Freon 22 (Cryoproducts Inc.) cooled by a bath of liquid nitrogen. Such tissue blocks could be stored in liquid nitrogen for many months without apparent morphological deterioration.

Semithin sections $(0.25$ to $0.5 \mu \mathrm{m})$ were cut using a Sorval MT2B microtome with an FTS cryo-attachment on a dry, $25-\mathrm{mm}$ glass knife at $-60^{\circ} \mathrm{C}$ to $-70^{\circ} \mathrm{C}$. The sections were transferred to precleaned, $13-\mathrm{mm}$ glass coverslips using a loop containing a saturated sucrose solution. Prior to staining, the coverslips were immersed in buffer for $20 \mathrm{~min}$ to wash away the sucrose, then in
$20 \mathrm{~mm}$ lysine to block free aldehyde groups, and finally in $2 \%(\mathrm{w} / \mathrm{v})$ bovine serum albumin (BSA; Pentex) to inhibit nonspecific binding of antibodies.

Immunofluorescence staining. All of the antibodies used in these studies have been described previously. Tissue sections on coverslips were incubated in A2B5 monoclonal antibody (Eisenbarth et al., 1979) (ascites fluid, diluted 1:100 in buffer containing $20 \%(\mathrm{v} / \mathrm{v})$ heatinactivated goat serum and $2 \% \mathrm{BSA}$ ), washed with five changes of buffer, and then incubated in rhodaminecoupled goat anti-mouse immunoglobulin (G anti-MIg$\mathrm{Rd}$, Cappel, diluted 1:100 as above). After washing, the sections were incubated in rabbit anti-GFAP (Pruss, 1979) (anti-GFAP, diluted 1:1000) or rabbit anti-210,000dalton neurofilament protein (Anderton et al., 1980) (anti-NF, diluted 1:100), washed, and incubated in fluorescein-coupled goat anti-rabbit immunoglobulin ( $G$ anti-RIg-Fl, Nordic, diluted 1:100), which had been absorbed with mouse immunoglobulin coupled to Sepharose 4B until it no longer cross-reacted with mouse immunoglobulin. All incubations were for $30 \mathrm{~min}$ at room temperature. After the final wash, sections were mounted in $14 \%$ (v/v) Gelvatol (Monsanto) and 30\% (v/v) glycerol in phosphate-buffered saline and examined in a Zeiss Universal incidence fluorescence microscope equipped with phase contrast, fluorescein, and rhodamine optics. Sections were photographed using Tri $\mathrm{X}$ film rated at 400 ASA.

In control experiments, semithin frozen sections were incubated in normal mouse ascites fluid (diluted 1:100) in place of the A2B5 antibody, and in normal rabbit serum (diluted 1:100) in place of the anti-GFAP or anti$\mathrm{NF}$ antiserum. No CNS staining was seen in such experiments, although the G anti-MIg-Rd weakly stained the meninges.

Immunogold electron microscopy of teased optic nerves. Adult rats were perfused for 5 min with $4 \%$ paraformaldehyde in buffer. Optic nerves were removed and immersed in the same fixative for $2 \mathrm{hr}$ at $4^{\circ} \mathrm{C}$, and then in $50 \%(\mathrm{v} / \mathrm{v})$ glycerol (Fisons) overnight at $4^{\circ} \mathrm{C}$. The nerves were then teased into small bundles and single fibers on poly-L-lysine-coated glass coverslips and fixed in place by immersion in $3 \%$ paraformaldehyde and $0.5 \%$ glutaraldehyde for $15 \mathrm{~min}$ at room temperature.

Prior to staining, the teased nerves were washed in buffer for $20 \mathrm{~min}$ followed by 30 -min incubations in 20 $\mathrm{mM}$ lysine and then $50 \%$ heat-inactivated goat serum. The nerves were then incubated in A2B5 antibody-containing ascites fluid or normal ascites fluid (diluted 1:100 in buffer containing 5\% BSA) overnight, washed with five changes of buffer for $5 \mathrm{~min}$ each, and then incubated in goat anti-MIg coupled to 20 - $\mathrm{nm}$ colloidal gold ( $\mathrm{G}$ antiMIg-Au; Janssen Pharmaceuticals; diluted 1:5 as above) for $30 \mathrm{~min}$ at room temperature. The nerves were then washed in three changes of buffer for $10 \mathrm{~min}$ each, further fixed in $1 \%$ glutaraldehyde for $30 \mathrm{~min}$ at room temperature followed by $1 \%$ osmium tetroxide for $1 \mathrm{hr}$ at $0^{\circ} \mathrm{C}$, and then dehydrated through graded ethanol and embedded in Epon 812 (Emscope). Thin sections were cut parallel to the substrate and stained with uranyl acetate and lead citrate before examination on a JFOL 100 CX11 electron microscope operating at $80 \mathrm{kV}$. 

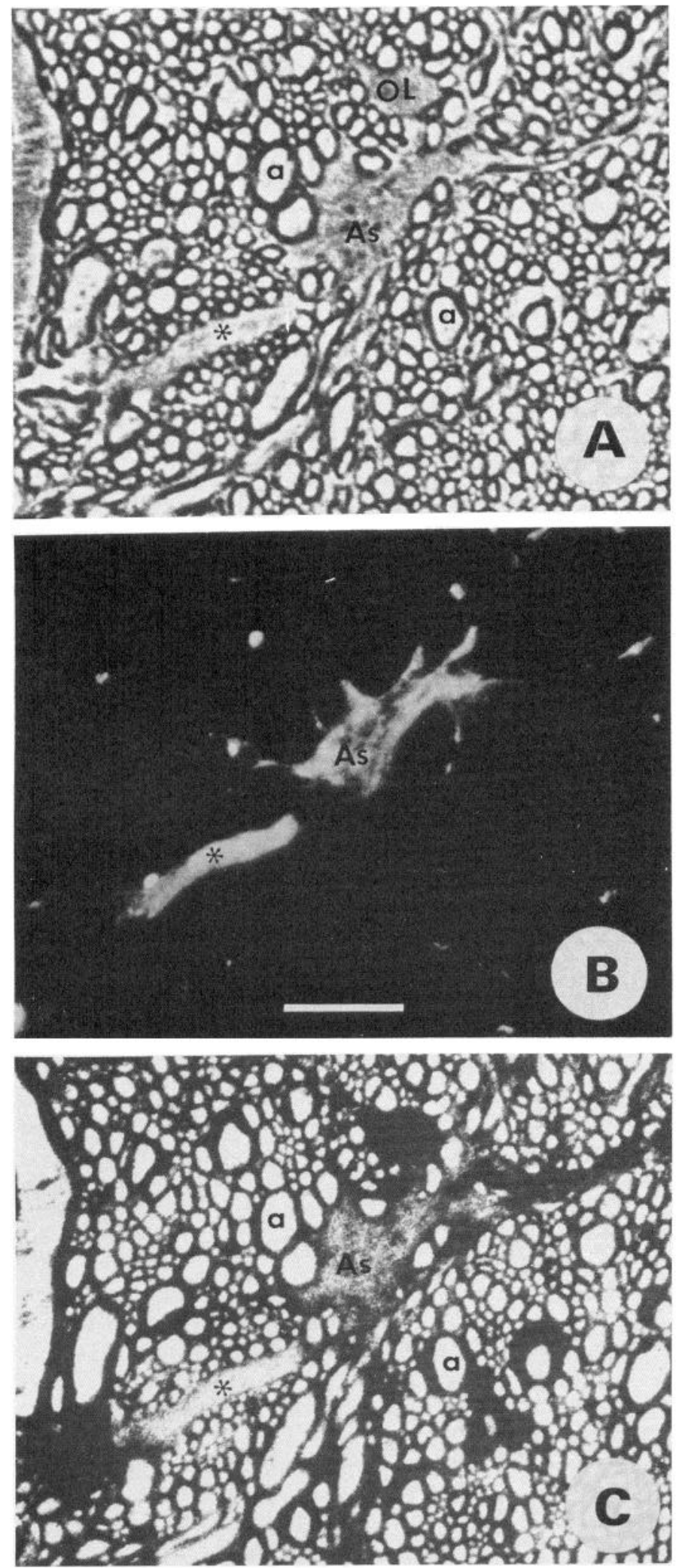

Figure 1. Transverse semithin frozen section from the central region of an adult optic nerve double labeled with anti-GFAP and A2B5 antibodies as described under "Materials and Methods." $A$, Phase contrast; $B$, fluorescein labeling with anti-GFAP antiserum; $C$, rhodamine labeling with A2B5 antibody. Note that only the astrocyte cell body $(A s)$ and process $(*)$ are labeled by anti-GFAP antibody $(B)$, whereas all of the axons (two of

\section{Results}

Adult optic nerve. When semithin, transverse frozen sections of adult optic nerve were studied by immunofluorescence using both A2B5 and anti-GFAP antibodies, almost all of the $\mathrm{GFAP}^{+}$astrocytes in the interior of the nerve were found to be A2B5 ${ }^{+}$(Fig. 1). Such astrocytes were intensely $\mathrm{GFAP}^{+}$, had processes that ran mainly transversely through the nerve (parallel to the plane of sectioning), and often ended on blood vessels. On the other hand, $\mathrm{GFAP}^{+}$astrocytes that formed the glial limiting membrane at the periphery of the nerve were not labeled by A2B5 antibody (Fig. 2). These cells, which also extended processes that ended on blood vessels, were only weakly $\mathrm{GFAP}^{+}$compared with the astrocytes that were located more centrally in the nerve. While $\mathrm{A} 2 \mathrm{~B}^{+}$, $\mathrm{GFAP}^{-}$cell bodies were only rarely seen in adult optic nerve, there were many $\mathrm{A}_{2} \mathrm{~B}^{-}, \mathrm{GFAP}^{-}$cells. The latter were usually small, round cells and were probably oligodendrocytes (Fig. 1). Endothelial cells and myelin sheaths were not stained by A2B5 or anti-GFAP antibodies, but some cells in the meninges surrounding the nerve were $\mathrm{A} 2 \mathrm{~B}^{+}$(not shown). When sections of adult optic nerve were double-labeled with A2B5 and anti-NF antibodies, all $\mathrm{NF}^{+}$axons were intensely A2B5 ${ }^{+}$(Fig. 3).

Since A2B5 antibody has been reported to bind to a polysialoganglioside (Eisenbarth et al., 1979), it was surprising to find that the staining of both axons and astrocytes by this monoclonal antibody appeared to be largely intracellular. Nonetheless, the staining seemed to be specific. (1) Although axons and some astrocytes were intensely stained by the antibody, many other cells within the nerve were not detectably stained. (2) When normal mouse ascites fluid or another mouse IgM monoclonal antibody (38/D7, which binds to cells in the peripheral but not central nervous system (Vulliamy et al., 1981)) were used instead of A2B5, no staining was seen. (3) When frozen sections were treated with Vibrio cholerae neuraminidase (Behring-Werke, Mannheim, 0.01 to $0.02 \mathrm{IU}$ in $0.1 \mathrm{M}$ phosphate buffer, $\mathrm{pH} 7.2$ for 30 min at $37^{\circ} \mathrm{C}$ ) prior to staining with the A2B5 antibody, the amount of labeling was greatly decreased.

To determine whether fibrous astrocytes in adult optic nerves expressed A2B5 antigen on their surface as well as intracellularly, teased nerves were studied by immunogold electron microscopy. In such preparations, A2B5 antibody labeled the surface of astrocyte processes and cell bodies, which were recognized by their characteristic bundles of glial filaments and paucity of microtubules (Fig. 4, $A$ and $B$ ). Myelinated axons were unlabeled except where the myelin had been stripped away, exposing the surface of the axon (Fig. $4 C$ ). Small dark cells with the morphological characteristics of oligodendrocytes were unlabeled. No significant labeling was seen when normal ascites fluid was used in place of A2B5 antibody-containing ascites fluid.

which are indicated with an $a$ in this and the next two figures), as well as the astrocyte cell body $(A s)$ and process (*), are labeled with A2B5 antibody $(C)$. The cell body $(O L)$, which is probably an oligodendrocyte, and the myelin sheaths surrounding the axons are not labeled by either antibody. Bar $=10 \mu \mathrm{m}$. 

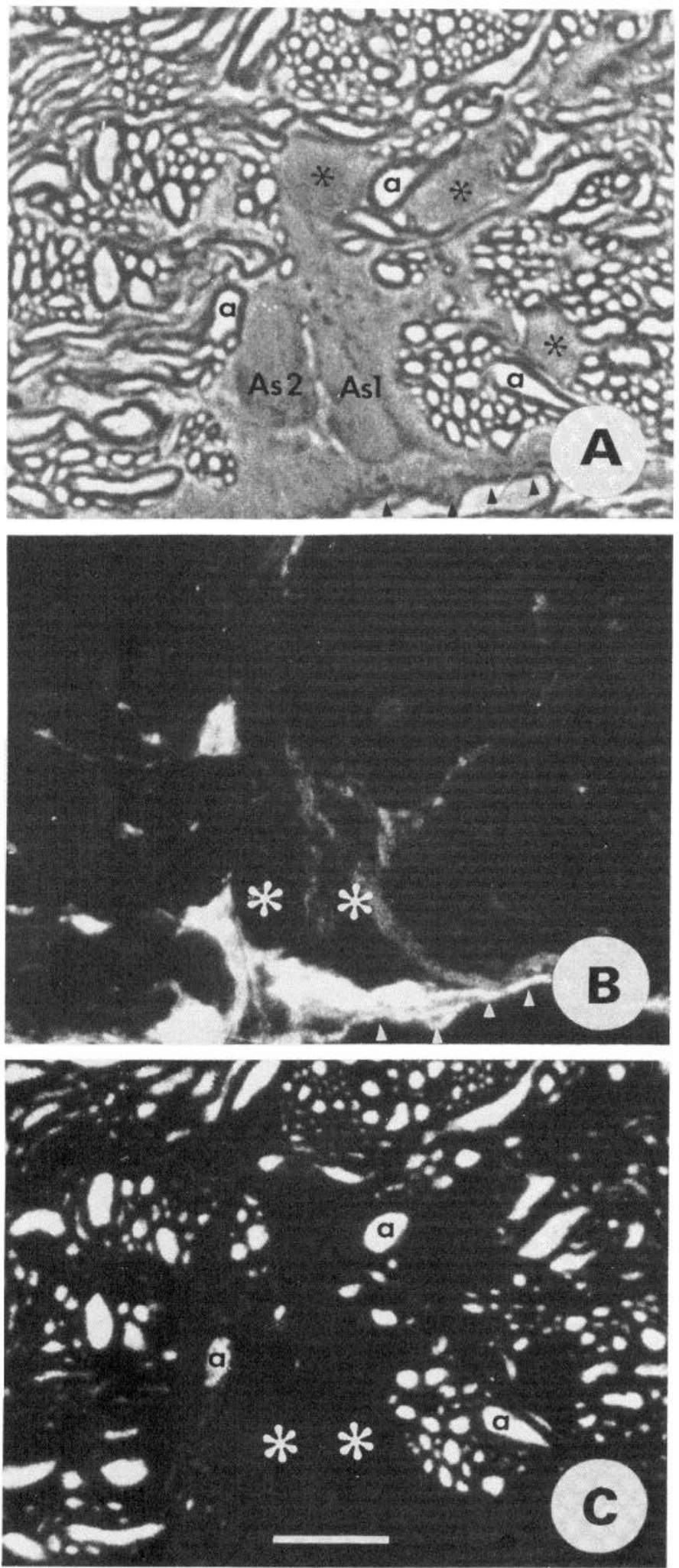

Figure 2. Transverse semithin frozen section from the periphery of an adult optic nerve, double labeled with anti-GFAP and A2B5 antibodies. $A$, Phase contrast; $B$, fluorescein labeling with anti-GFAP antiserum; $C$, rhodamine labeling with A2B5 antibody. The two astrocyte cell bodies ( $A s 1$ and $A s 2$ in $A$, white asterisks in $B$ and $C$ ) and the astrocyte processes forming the glial limiting membrane (arrowheads) are $\mathrm{GFAP}^{+}(B)$ but $\mathrm{A}^{2} \mathrm{B5}^{-}(C)$. The exposure time in $B$ was much longer than in Figure $1 B$, as the anti-GFAP labeling was much weaker than
Developing optic nerve. When semithin frozen sections of 1- or 7-day optic nerve were studied by immunofluorescence, all of the $\mathrm{NF}^{+}$axons were found to be $\mathrm{A} 2 \mathrm{~B}^{+}$, just as in the adult (not shown). However, all of the $\mathrm{GFAP}^{+}$astrocytes were A2B5- (Fig. 5). Most of the astrocytes were located toward the periphery of the nerve and had processes that contributed to the glial limiting membrane. These cells stained more intensely with antiGFAP antiserum than did A2B5 ${ }^{-}$astrocytes in adult optic nerves. Moreover, unlike the case in adult optic nerve, there were many $\mathrm{A} 2 \mathrm{~B}^{+}, \mathrm{GFAP}^{-}$cell bodies in 1and 7-day nerves. Most of these were small, non-processbearing cells situated toward the center of the nerve. There were also many cells that were not labeled by either A2B5 or anti-GFAP antibodies.

In 15-day optic nerve a small proportion of $\mathrm{GFAP}^{+}$ astrocytes were $\mathrm{A} 2 \mathrm{B5}^{+}$, whereas in 28-day optic nerve more than half of the astrocytes were A2B $5^{+}$. All of the $\mathrm{A} 2 \mathrm{~B}^{+}$astrocytes in 15- and 28-day optic nerve were located in the interior of the nerve; the astrocytes at the periphery of the nerve were always A2B5 ${ }^{-}$.

Adult cerebral cortex. When semithin, coronal, frozen sections of adult cerebral cortex were double-labeled with A2B5 and anti-GFAP or anti-NF antibodies, all of the $\mathrm{NF}^{+}$axons were $\mathrm{A} 2 \mathrm{B5}^{+}$. Relatively few $\mathrm{GFAP}^{+}$astrocytes were seen and most of them contributed to the glial limiting membrane and stained relatively weakly with anti-GFAP antibody (Fig. 6). Such cells were A2B5 ${ }^{-}$. Only occasional GFAP ${ }^{+}$cells in the deeper regions of the cortex were $\mathrm{A} 2 \mathrm{~B}^{+}$.

\section{Discussion}

By studying semithin frozen sections of adult and developing rat CNS by immunofluorescence we have extended our previous observations on type 1 and type 2 astrocytes in vitro (Raff et al., 1983a) in several ways. Most importantly, we have found both $\mathrm{A} 2 \mathrm{~B}^{+}$and $\mathrm{A} 2 \mathrm{~B} 5^{-}$ astrocytes in adult CNS, making it unlikely that these two astrocyte phenotypes represent different stages in the maturation of a single cell type. Since the great majority of astrocytes in white matter (optic nerve) are labeled intensely by both anti-GFAP and A2B5 antibodies, whereas most of those in gray matter (cerebral cortex) are labeled relatively weakly by anti-GFAP and not at all by A2B 5 antibodies, it seems likely that the A2B5 ${ }^{+}$ and $\mathrm{A} 2 \mathrm{~B} 5^{-}$astrocytes in these regions of $\mathrm{CNS}$ correspond to fibrous and protoplasmic astrocytes, respectively.

Since the optic nerve is composed entirely of white matter, it generally has been thought that its astrocytes are almost exclusively of the fibrous type, and this has been assumed in interpreting studies on astrocyte development in the optic nerve (Vaughn and Peters, 1967; Skoff et al., 1976a, b). However, our previous observations on astrocyte heterogeneity in cultures of developing optic nerve were difficult to reconcile with this view (Raff et al., 1983a), and our present findings provide firm evidence that there are at least two classes of astrocytes

in Figure $1 B$. Note that, while the axons are $\mathrm{A}^{2} \mathrm{~B}^{+}, \mathrm{GFAP}^{-}$, there are three cell bodies ( $*$ in $A$ ), which may be oligodendrocytes, that are not labeled by either antibody. Bar $=10 \mu \mathrm{m}$. 

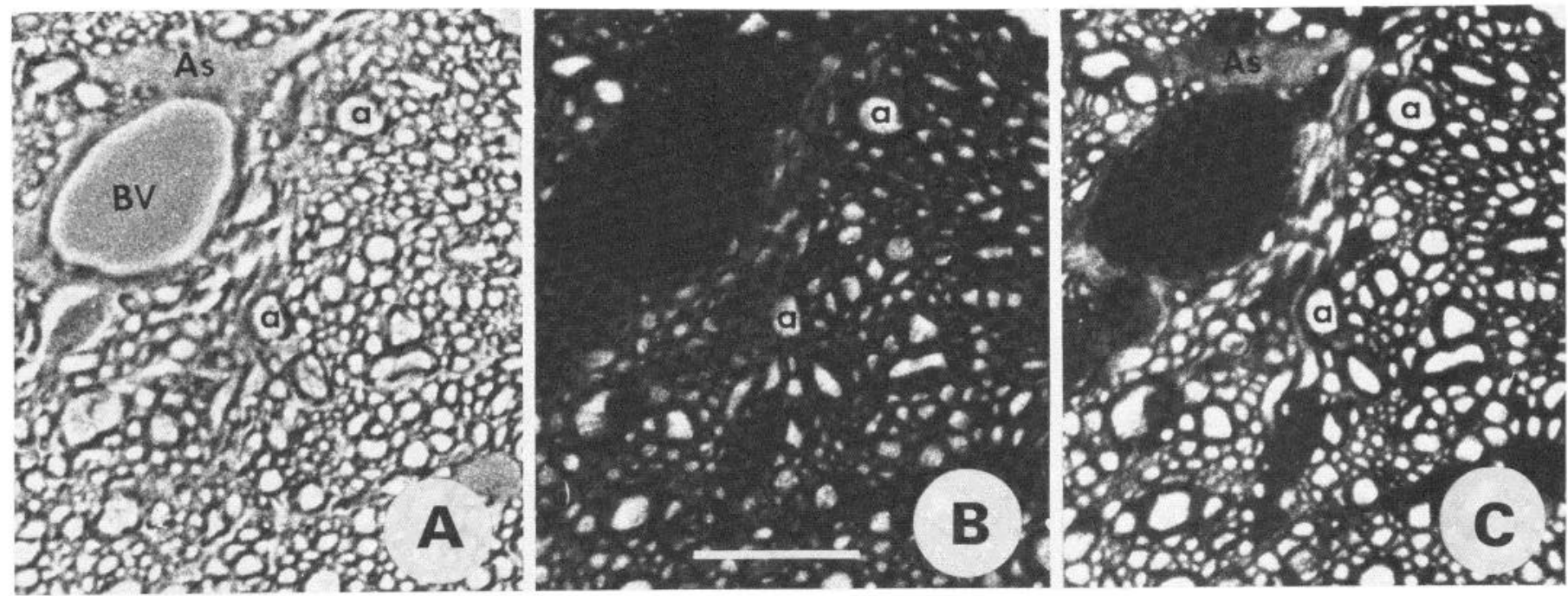

Figure 3. Transverse semithin frozen section from the central region of an adult optic nerve double labeled with antineurofilament (NF) and A2B5 antibodies. $A$, Phase contrast; $B$, fluorescein labeling with anti-NF antiserum; $C$, rhodamine labeling with A2B5 antibody. All of the $\mathrm{NF}^{+}$axons $(B)$ are $\mathrm{A}_{2} \mathrm{~B} 5^{+}(C)$, while the presumptive astrocyte processes surrounding the blood vessel $(B V)$ are $\mathrm{A}_{2} \mathrm{~B}^{+}(C)$ but $\mathrm{NF}^{-}(B) . B a r=20 \mu \mathrm{m}$.

in the adult rat optic nerve. While most of the astrocytes in the interior of the adult optic nerve have the antigenic phenotype of fibrous astrocytes, being intensely GFAP ${ }^{+}$ and $\mathrm{A} 2 \mathrm{~B} 5^{+}$, those forming the glial limiting membrane at the periphery of the nerve have the antigenic phenotype of protoplasmic astrocytes, being only weakly $\mathrm{GFAP}^{+}$and $\mathrm{A} 2 \mathrm{~B}^{-}{ }^{-}$. The latter cells are probably equivalent to the protoplasmic astrocytes that form the glial limiting membrane in the cerebral cortex. Whereas initially it seemed prudent to use the tentative "type 1-type 2 " terminology to refer to the two astrocyte phenotypes found in cultures of developing optic nerve (Raff et al., $1983 \mathrm{a}, \mathrm{b})$, it now seems appropriate to use the "protoplasmic-fibrous" terminology for optic nerve astrocytes in culture as well as in situ.

Whereas protoplasmic astrocytes in tissue sections stain relatively weakly with anti-GFAP antibodies (Bignami et al., 1972; Ludwin et al., 1976) and contain relatively few glial filaments (Peters et al., 1976), those in culture generally stain intensely with anti-GFAP antibodies and contain many bundles of glial filaments $(R$. $H$. Miller, unpublished observations). One explanation for this discrepancy may be that the protoplasmic astrocytes studied in culture are usually from neonatal animals, whereas those studied in tissue sections are usually from mature animals. Our finding that the protoplasmic astrocytes at the periphery of the optic nerve stained more intensely with anti-GFAP antibodies in 7-day than in adult animals suggests that at least some protoplasmic astrocytes may express less GFAP and fewer glial filaments as they mature. Another explanation may be that protoplasmic astrocytes are induced to make more GFAP and glial filaments when they are dissociated from the CNS and grown in culture. Such cells may be equivalent to reactive astrocytes, most of which contain large amounts of GFAP (Bignami and Dahl, 1974) and many glial filaments (Maxwell and Kruger, 1965) and have the cell surface properties of protoplasmic astrocytes (Raff et al., 1983a).
Our observations on frozen sections of developing optic nerves largely confirm our previous results on cell suspensions prepared from such nerves (Raff et al., 1983a). In both cases, we find large numbers of $\mathrm{A}_{2} \mathrm{~B}^{+}, \mathrm{GFAP}^{-}$ cells, while the great majority of $\mathrm{GFAP}^{+}$astrocytes are A2B5 ${ }^{-}$. This contrasts with the findings in frozen sections of adult optic nerve where there are very few $\mathrm{A} 2 \mathrm{~B} 5^{+}$, GFAP $^{-}$cells and the great majority of astrocytes are $\mathrm{A}^{2} \mathrm{~B}^{+}$. From studies of tissue sections it is not possible to determine unambiguously whether the large numbers of $\mathrm{A} 2 \mathrm{~B}^{+}, \mathrm{GFAP}^{+}$fibrous astrocytes in adult optic nerve develop from $\mathrm{A} 2 \mathrm{B5}^{-}, \mathrm{GFAP}^{+}$astrocytes, which acquire the A2B5 antigen with age, or from $\mathrm{A} 2 \mathrm{~B} 5^{+}, \mathrm{GFAP}^{-}$cells, which acquire GFAP with age. However, our two previous studies strongly support the latter view. First, we found that $\mathrm{A}^{2} \mathrm{~B}^{-}, \mathrm{GFAP}^{+}$(protoplasmic) astrocytes do not acquire A2B5 even after weeks in culture; although these cells can be induced to adopt a fibrous astrocyte morphology, they still do not bind tetanus toxin or A2B5 antibody (Raff et al., 1983a). Second, by using antibodies to identify and manipulate glial cells and their precursors in vitro, we showed that $\mathrm{A} 2 \mathrm{~B} 5^{+}$fibrous astrocytes develop in culture from $\mathrm{A}_{2} \mathrm{~B}^{+}$, $\mathrm{GFAP}^{-}$progenitor cells, which can differentiate into either oligodendrocytes or fibrous astrocytes depending on the culture conditions (Raff et al., 1983b). Taken together, our results in vivo and in vitro suggest that protoplasmic and fibrous astrocytes are distinct classes of glial cells that differ in their biochemistry and developmental history, as well as in their morphology and location in the CNS.

An unexplained finding in the present study was that the immunofluorescence staining of axons and fibrous astrocytes with A2B5 antibody in frozen sections appeared to be largely intracellular, although we were able to demonstrate surface labeling of these structures by immunogold electron microscopy of teased optic nerves. Intracellular staining with A2B5 antibody was also observed in frozen sections of retina by Eisenbarth et al. (1979) in their initial description of the A2B5 antibody 


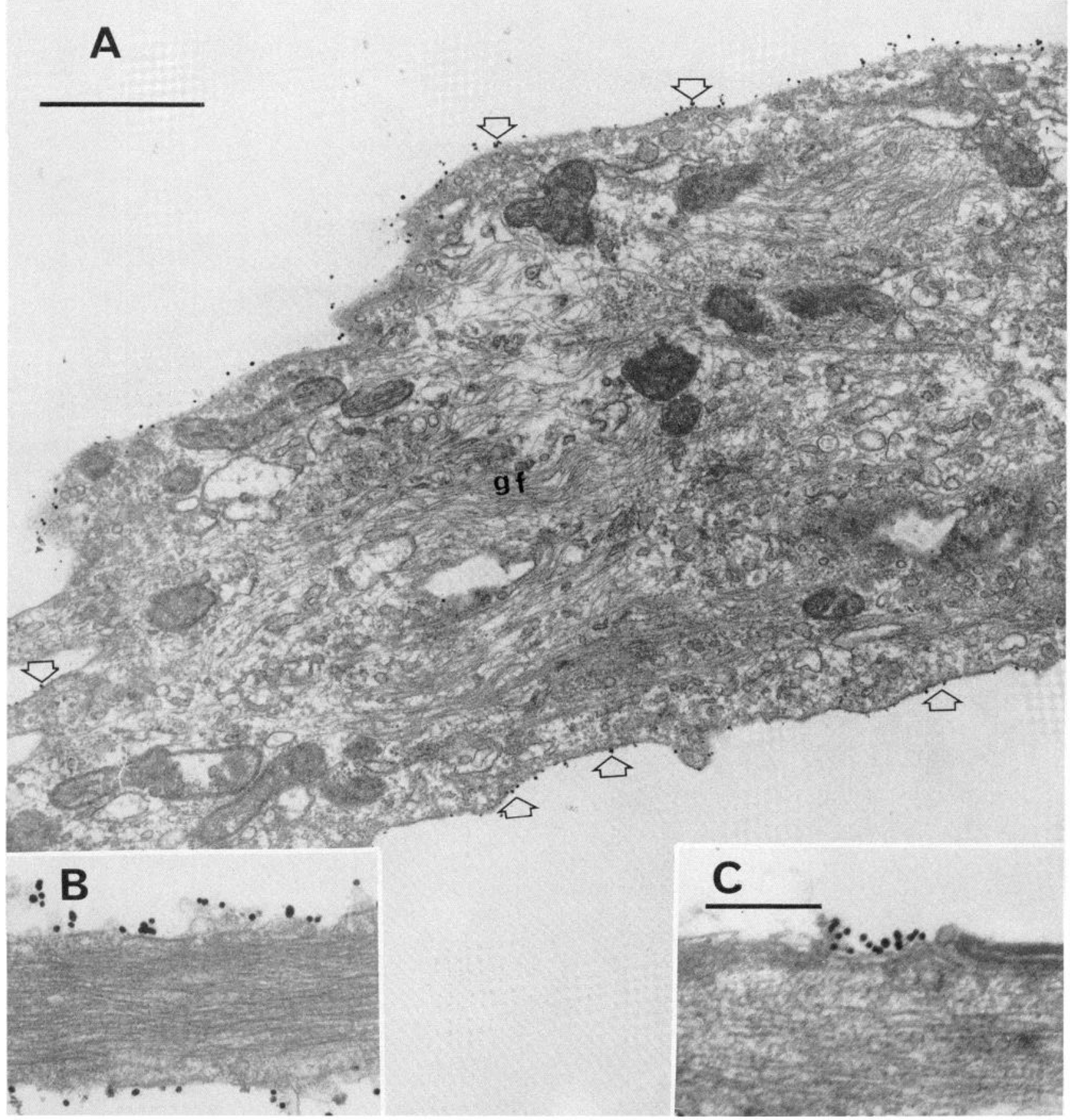

Figure 4. Electron micrographs of immunogold-labeled adult optic nerve. The nerve was fixed, teased, and labeled with A2B5 antibody followed by G anti-MIg-Au as described under "Materials and Methods." A, Longitudinal section of an astrocyte process that is labeled on its surface by the A2B5 antibody (arrows). Note the presence of bundles of glial filaments ( $g f$ ) and the paucity of microtubules in the cytoplasm. Bar $=10 \mu \mathrm{m}$. B, Longitudinal section through a thin astrocyte process, which is packed with glial filaments and is labeled on its surface by the A2B5 antibody. $C$, Longitudinal section through a myelinated axon. Note that the A2B5 antibody has bound to the surface of the axon where the overlying myelin has been stripped away. $B a r$ for $B$ and $C=$ $3 \mu \mathrm{m}$.

and has been seen with other antibodies that recognize glycolipid antigens (Marcus and Janis, 1970). In none of these cases has the intracellular location of the antigen been identified. Since the cell type specificity of the intracellular labeling with A2B5 antibody on frozen sections is the same as when surface labeling is observed on cells in teased optic nerves or on living cells in vitro, it seems likely that the antibody is binding to the same oligosaccharide inside the cell as on the cell surface; the sensitivity of both surface and intracellular antigens to neuraminidase is consistent with this view. However, our observations do not address the question of whether the intracellular antigen is a glycolipid, glycoprotein, or both.

Although our findings suggest that fibrous and protoplasmic astrocytes are biochemically and developmentally distinct, they do not imply that these are the 

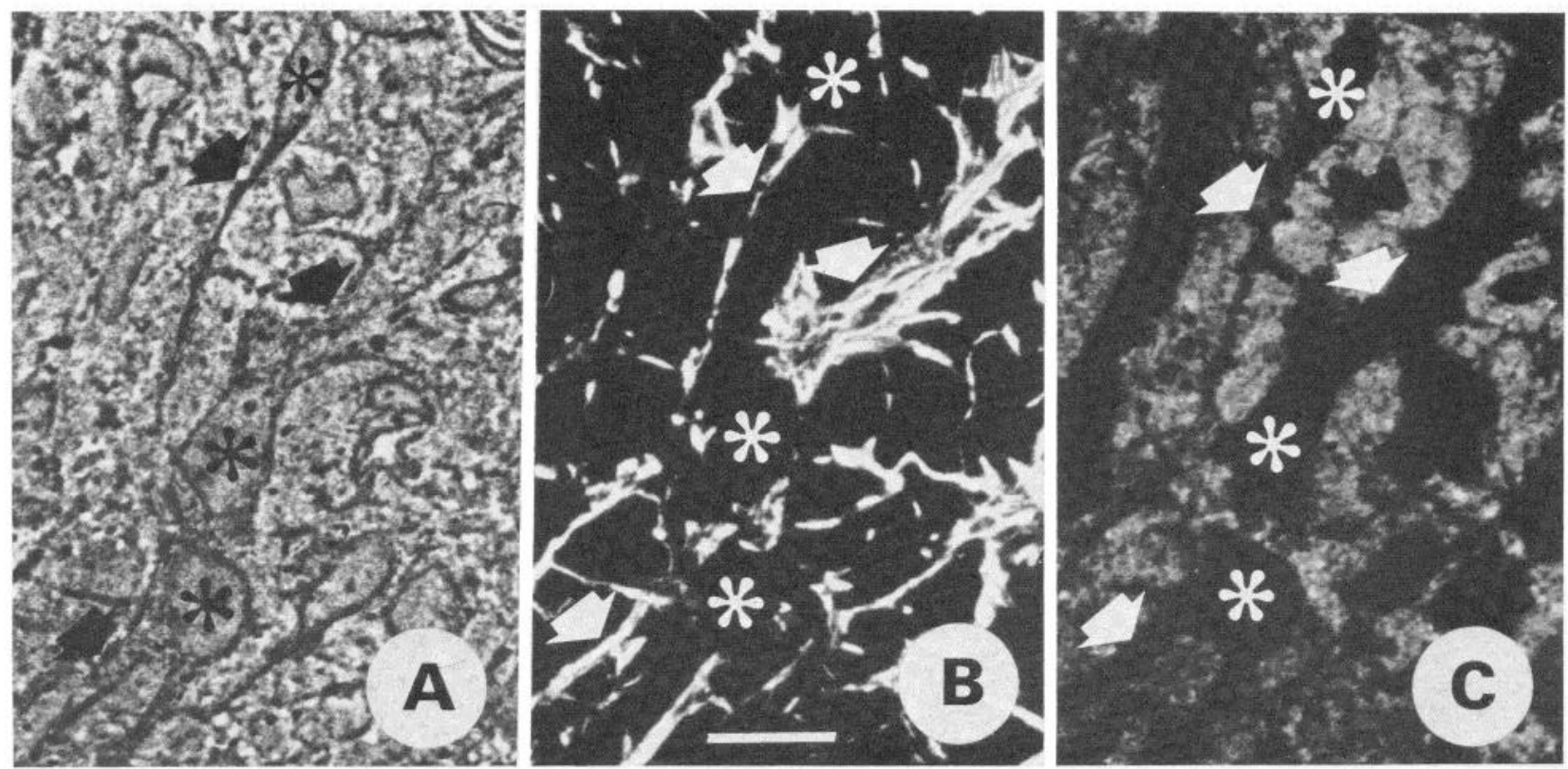

Figure 5. Transverse semithin frozen section from the central region of a 7-day optic nerve double labeled with anti-GFAP and A2B5 antibodies. $A$, Phase contrast; $B$, fluorescein labeling with anti-GFAP antiserum; $C$, rhodamine labeling with A2B5 antibody. The processes (arrows) but not the cell bodies $\left(^{*}\right)$ of three astrocytes are $\mathrm{GFAP}^{+}(B)$. While astrocyte cell bodies and processes are $\mathrm{A}^{2} \mathrm{~B}^{-}$, the axons in the nerve (which at this age are too small to be resolved individually) are $\mathrm{A} 2 \mathrm{~B} 5^{+}(C)$. $B a r=$ $10 \mu \mathrm{m}$.
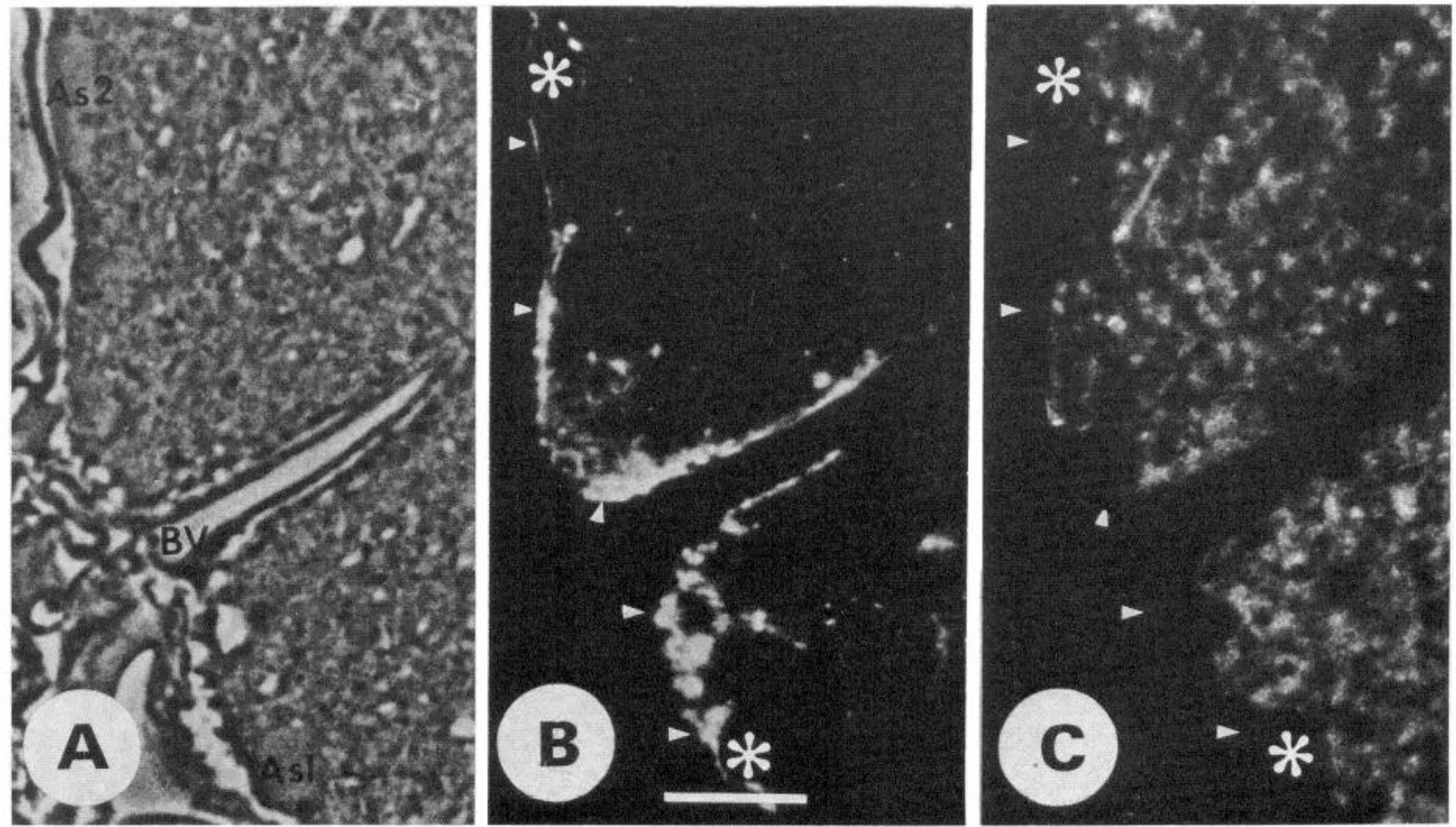

Figure 6. Coronal semithin frozen section of adult cerebral cortex plus overlying meninges double-labeled with anti-GFAP and A2B5 antibodies. $A$, phase contrast; $B$, fluorescein labeling with anti-GFAP antiserum; $C$, rhodamine labeling with A2B5 antibody. Two astrocyte cell bodies ( $A s 1$ and $A s 2$ in $A$, white asterisks in $B$ and $C$ ) and the astrocyte processes forming the glial limiting membrane and surrounding the blood vessel $(B V)$ are $\mathrm{GFAP}^{+}(B)$ but A2B5 $(C)$. The A2B5 labeling appears to be restricted to cortical neurons and their processes $(C)$. Bar $=10 \mu \mathrm{m}$.

only classes of astrocytes in the CNS, or that each of these classes is homogeneous. Anatomists have distinguished other types of astrocytes or astrocyte-like cells, including Bergmann glia in the cerebellum and radial glial cells in developing CNS, and it seems likely that, with time, these and the other classes of astrocytes will be progressively subdivided into molecularly distinct subclasses. However, until one can establish the functions of astrocytes, the biological significance of astrocyte heterogeneity will remain uncertain. 


\section{References}

Anderton, B. H., R. Thorpe, J. Cohen, S. Selvendran, and P. Woodhams (1980) Specific neuronal localization by immunofluorescence of $10 \mathrm{~nm}$ filament polypeptides. J. Neurocytol. 9: 835-844.

Bignami, A., and D. Dahl (1974) Astrocyte-specific protein and radial glia in the cerebral cortex of new born rat. Nature 252: 55-56.

Bignami, A., L. F. Eng, D. Dahl, and C. T. Uyeda (1972) Localization of the glial fibrillary acidic protein in astrocytes by immunofluorescence. Brain Res. 43: 429-435.

Cajal, S. R. (1913) Sobre un nuevo proceder de impregnación de la neuroglia y sus resultados en los centros nerviosos del hombre y animales. Trab. Lab. Invest. Biol. Univ. Madrid 11: $219-237$.

Eisenbarth, G. S., F. S. Walsh, and M. Nirenberg (1979) Monoclonal antibody to a plasma membrane antigen of neurons. Proc. Natl. Acad. Sci. U. S. A. 76: 4913-4917.

Ludwin, S. K., J. C. Kosek, and L. F. Eng (1976) The topographical distribution of S-100 and GFA proteins in the adult brain: An immunohistochemical study using horseradish peroxidase-labelled antibodies. J. Comp. Neurol. 165: 197-208.

Marcus, D. M., and R. Janis (1970) Localization of glycosphingolipids in human tissues by immunofluorescence. J. Immunol. 104: 1530-1539.

Maxwell, D. S., and L. Kruger (1965) The fine strucutre of astrocytes in the cerebral cortex and their response to focal injury produced by heavy ionizing particles. J. Cell Biol. 25: 141-157.

Mori, S., and C. P. Leblond (1969) Electron microscopic features and proliferation of astrocytes in the corpus callosum of the rat. J. Comp. Neurol. 137: 197-226.

Peters, A., S. L. Palay, and H. de F. Webster (1976) The Fine Structure of the Nervous System: The Neurons and Support- ing Cells, pp. 242-244, W. B. Saunders Co., Philadelphia.

Pruss, R. (1979) Thy-1 antigen on astrocytes in long-term cultures of rat central nervous system. Nature 280:688-690.

Raff, M. C., E. R. Abney, J. Cohen, R. Lindsay, and M. Noble (1983a) Two types of astrocytes in cultures of developing rat white matter: Differences in morphology, surface gangliosides, and growth characteristics. J. Neurosci. 3: 1289-1300.

Raff, M. C., R. H. Miller, and M. Noble (1983b) A glial progenitor cell that develops in vitro into an astrocyte or an oligodendrocyte depending on the culture medium. Nature 303: 390-396.

Schachner, M., E. T. Hedley-Whyte, D. W. Hsu, G. Schoonmaker, and A. Bignami (1977) Ultrastructural localization of glial fibrillary acidic protein in mouse cerebellum by immunoperoxidase labelling. J. Cell Biol. 75: 67-73.

Skoff, R., D. Price, and A. Stocks (1967a) Electron microscopic autoradiographic studies of gliogenesis in rat optic nerve. I. Cell proliferation. J. Comp. Neurol. 169: 291-312.

Skoff, R., D. Price, and A. Stocks (1976b) Electron microscopic autoradiographic studies of gliogenesis in rat optic nerve. II. Time of origin. J. Comp. Neurol. 169: 313-333.

Tokuyasu, K. T. (1973) A technique for ultracryotomy of cell suspensions and tissues. J. Cell Biol. 37: 551-565.

Van Heyningen, W. E. (1963) The fixation of tetanus toxin, strychnine, serotonin and other substances by gangliosides. J. Gen. Microbiol. 3: 375-387.

Vaughn, J. E., and D. C. Pease (1967) Electron microscopy of classically stained astrocytes. J. Comp. Neurol. 131: 143-153.

Vaughn, J. E., and A. Peters (1967) Electron microscopy of the early postnatal development of fibrous astrocytes. Am. J. Anat. 121: 131-152.

Vulliamy, T., S. Rattray, and R. Mirsky (1981) Cell-surface antigen distinguishes sensory and autonomic peripheral neurons from central neurons. Nature 291: 418-420. 